Review Article

\title{
Infliximab management complex regional pain syndrome
}

\begin{abstract}
The role of inflammatory mechanisms in the pathogenesis of CRPS is important and that makes it a target that must meet every day. Infliximab is a molecule that has been used in the management of CRPS and published case reports and two studies showing improvement in range of motion, differences in temperature and decrease pain persist even phenomena of peripheral and Central after a few months; as same adverse reactions have been reported including appearance of CRPS in patients who received other necessary conditions and use with caution. The information is so far low level of evidence for such studies from which it was generated so it is necessary to have higher quality studies to obtain more solid conclusions.
\end{abstract}

\section{Summary}

The complex regional pain syndrome (CRPS) is a variety of painful conditions of regional finding, after an injury, with distal predominance with symptoms that exceed in magnitude and duration the expected clinical course, with motor impairment and progressive course. Within the pathophysiology of complex regional pain syndrome multiple factors that produce and therefore proposed treatments are evaluated current evidence is recognized. One mechanism proposed that may contribute to the occurrence of CRPS is a tumor necrosis factor, $\mathrm{TNFa}$, and a therapeutic proposal is the use of monoclonal antibody TNFa inhibitor, infliximab. The aim is to review aspects of diagnosis, pathophysiology and current evidence on the use of infliximab in CRPS.

Keywords: complex regional pain syndrome, diagnosis, pathophysiology, infliximab
Volume 3 Issue I - 2015

\section{Edmundo Gonima}

Anestesiólogo Algesiólogo. Intervencionista En Dolor, USA

Correspondence: Edmundo Gonima,Anestesiólogo Algesiólogo. Intervencionista En Dolor, USA, Tel 31743 I5792, Email edmundorama@gmail.com

Received: June 10,2015 | Published: September 22, 2015

\section{Introduction}

The complex regional pain syndrome (CRPS) has several definitions from the start made by Claude Bernard in 1851 who mentioned a painful syndrome associated with dysfunction of the sympathetic nervous system to reach consensus in Orlando in 1994 and later in Budapest in $2007^{1}$ to establish revised procedures for the diagnosis of complex regional pain syndrome with a sensitivity of $85 \%$ and specificity of $69 \%{ }^{2}$ criteria. These criteria indicate that the complex regional pain syndrome is characterized by a continuous regional pain, evoked or spontaneous; it's seemingly disproportionate in time or degree of the usual course of any trauma or injury. The pain is regional, not a specific territory or dermatome nervous, and usually has a distal predominance of abnormal findings sensory, motor, vasomotor, and trophic sudomotor. This syndrome has a variable progression over time. ${ }^{1}$

\section{Clinical diagnostic criteria' are as follows}

1. Continuing pain that is disproportionate to any inciting event.

2. A symptom in at least three of the following four categories.

I. Sensory: Report hyperalgesia and/or allodynia,

II. Vasomotor: Report asymmetry in temperature and/or changes in skin color.

III. Sudomotor/edema: edema Report and/or changes in sweating.

IV. Motor/trophic: Report decreased ranges of motion and/or motor dysfunction and/or trophic changes.

1. A sign in two or more of the following three categories:

a. Sensory: evidence of hyperalgesia and / or allodynia. b. Vasomotor: Evidence of asymmetry in temperature and/or changes in skin color.

c. Sudomotor/edema: evidence of edema or sweating changes.

d. Motor/trophic: evidence of decreased range of motion and/or motor dysfunction and / or trophic changes.

2. There is no other diagnosis that better explains the symptoms and signs.

\section{Epidemiology}

Differences in incidence in several studies found Moss et al. ${ }^{3}$ estimate the incidence 26.2 per 100,000 inhabitants per year, ${ }^{3}$ while the reports Sandroni 5.6 per 100,000 inhabitants per year ${ }^{4}$ is related to trauma in $65 \%$ of cases. ${ }^{5}$ It is more common in women; you may follow any type of trauma, even spontaneously, and the most common trigger events are surgery, fractures, crush traumas and sprains; It is the most common type I, that is, the nerve damage has not demonstrated. In Colombia in the Valle del Cauca an incidence of 1.1 per 100,000 population, predominantly in upper limbs, male female ratio was found $1: 1.1$, average age $44,56 \%$ commitment of law and bilateral $5 \%$ side; within symptoms sweating changes in $96 \%, 92 \%$ and color temperature $90 \%$. $^{6,7}$

\section{Pathophysiology}

The complex regional pain syndrome is a painful chronic neuropathic disorder with major regional characteristics and develops in a limb after tissue trauma. ${ }^{8}$ In addition to the classic features of neuropathic pain, a burning pain, hyperalgesia and allodynia, presents edema and changes suggestive of regional commitment, abnormal sweating, skin color and temperature in the affected region and trophic 
changes in skin, hair and nails, and impaired motor function has been subdivided into type I and type II if presents no documented or nerve injury, but the signs and symptoms are similar and there is no evidence that any difference in the pathophysiological mechanisms or in response treatment. ${ }^{8}$ The patient experiences severe pain greatest functional disability and psychological suffering. CRPS has no definitive medical treatment and clinical trials have failed to support the efficacy of treatments commonly used; other treatments used are spinal cord stimulation and intrathecal administration systems drugs, which significantly increase the cost of managing these patients. These therapeutic failures are due in part to insufficient knowledge of the pathophysiological mechanisms involved. Multiple mechanisms are involved in their genesis; It is not only a peripheral pain mediated by the sympathetic system but is a central nervous system disease because there are changes in the somatosensory system and the bilateral sympathetic symptoms exist in one end. ${ }^{8}$ Eight have been proposed mechanisms that may contribute to the onset of CRPS.

Alteration of cutaneous innervation after trauma: It has been observed in animal models and in patients with CRPS type I decreased density of fibers $\mathrm{C}$ and $29 \%$ Ad skin biopsies limb pain and abnormal innervation around of hair follicles and sweat glands., ${ }^{910}$ Animal studies indicate that these findings may be caused by nerve injury in humans but that relationship is not clear.

Central sensitization: severe or persistent income after tissue damage or injury nociceptive nerve impulses causes an increase in the excitability of nociceptive neurons in the spinal cord, what we call Central sensitization. This phenomenon is mediated by the release of neuropeptides such as substance $\mathrm{P}$ and bradykinin, and excitatory amino acid glutamate acting at the N-methyl D-aspartate NMDA receptor; the result is the appearance of allodynia and hyperalgesia. ${ }^{11}$ It is not known if central sensitization occurs before, simultaneously or after the onset of other symptoms and signs of CRPS; there is the possibility that higher previous pain has increased central sensitization and onset of CRPS.

Peripheral sensitization: After tissue trauma peripheral sensitization is generated; primary afferent fibers traumatized area pronociceptor release neuropeptides like substance $\mathrm{P}$ and bradykinin that increase the activation of nociceptors and decreases the activation threshold for thermal and mechanical stimuli that contribute to the onset of hyperalgesia and allodynia; ${ }^{12}$ it is likely that these arise from early stages of CRPS

Altered functioning of the central nervous system: It is assumed that the cold and cyanotic extremity is due to vasoconstriction is a reflection of increased activity of the sympathetic nervous system and that the pain was sympathetically maintained. Animal studies indicate that after the nerve trauma adrenergic receptors are expressed in nociceptive fibers, a mechanism by which the sympathetic nervous system can generate nociceptive signals. ${ }^{13}$ The expression of these receptors contribute to sympathetic-afferent coupling has been demonstrated in humans, increasing the spontaneous pain and hyperalgesia $22 \%$ between 27 and $42 \%$; This component of sympathetically mediated pain may decrease over time. Not necessarily over-activity of the sympathetic system is responsible. In a prospective study in humans reduced sympathetic activity was observed before the onset of CRPS, both the affected and the unaffected, suggesting systemic alterations in sympathetic regulation rapidly after injury ${ }^{14}$ end; this change is a higher risk of CRPS. This reduction in sympathetic activity is associated with the initial stage where the patient is warm and red tip; othe studies indicate that in chronic phase occurs decrease in endothelium-dependent vasodilation and altered levels of endothelin, nitric oxide and nitric oxide synthase. ${ }^{10}$

Role of circulating catecholamines has been observed decreased levels of catecholamines in the affected side, implying a decrease in sympathetic action at local level: in CRPS is associated with chronic vasoconstriction; in the acute stage are decreased but then compensates receptors and increased receptor occurs which results in high sensitivity to circulating catecholamines, and sweating vasoconstriction in chronic phase; ${ }^{15}$ local hypoxia contributes to the observed trophic changes.

Brain plasticity: Several neuroimaging studies suggest a reorganization of somatotopic map consisting of a reduction in size of the representation of the affected in the somatosensory cortex ${ }^{16}$ and returning to normal after successful treatment tip ${ }^{17}$ suggesting that reflect brain plasticity which occurs during the development of CRPS. The degree of reorganization somatotopic correlates with pain intensity and degree of hyperalgesia. Neuroimaging studies suggest altered activity in sensory, motor and emotional regions; other studies suggest atrophy of gray matter in the insula and ventromedial prefrontal cortex.

Genetic factors: Relatives of patients with CRPS CRPS are more often spontaneous and at younger ages; ${ }^{18} \mathrm{SDRC}$ is no association between mitochondrial disorders in Young It found that some genes of major histocompatibility complex (HLA) are found more in CRPS and other less frequently; HLA-B62 and HLA-DQ8 alleles were significantly associated with CRPS ${ }^{19}$ and TNF2 allele is most commonly found in CRPS hot phase.

Psychological factors: Factors such as emotional stress may be associated with increased activity of catecholamines; a greater degree of depression increases the intensity of pain in CRPS and with higher levels of epinephrine and norepinephrine. ${ }^{20}$ Also psychological stress has been associated with impaired immune function which may impact on inflammatory cytokines that contribute to the SDRC; other studies suggest that patients with CRPS are more emotional stress.

Inflammatory factors: Inflammatory mechanisms may contribute to the SDRC, at least in its initial phase. Inflammation can arise from classical inflammatory and neurogenic inflammation mechanisms. Classic inflammatory mechanisms in lymphocytes and mast cells secrete proinflammatory cytokines such as interleukin 1B, 2 and 6 , and tumor necrosis factor (TNFa) which can produce localized edema and plasma extravasation. ${ }^{12}$ Neurogenic inflammation occurs because nociceptive fibers release proinflammatory cytokines and neuropeptides as substance $\mathrm{P}$, related peptide and calcitonin gene bradykinin, which produces vasodilation and plasma extravasation, warmth, redness and edema, osteoporosis, hair growth, sweating and awareness peripheral. ${ }^{21}$

CRPS patients have a large increase in proinflammatory cytokines in cerebrospinal fluid and plasma and decreased levels of antiinflammatory cytokines. ${ }^{22}$ CRPS patients with hyperalgesia have higher levels of TNFa. This cytokine is important because it has direct pronociceptive actions and for inducing production of other cytokines involved in inflammation. Other findings include increased gene related peptide calcitonin, which produces vasodilation, edema and increased sweating; plus increasing concentrations of bradykinin, substance $\mathrm{P}$ and also a low ability to inactivate the substance $\mathrm{P}^{23}$ Treatment and Prognosis. Currently physiotherapy, occupational therapy, corticosteroids early in short cycle and bisphosphonates in the first six months have level I evidence and recommendation A. Moreover 
sympathetic blocks have level III evidence and recommendation C and neurostimulation in advanced stages It has evidence level II and recommendation $\mathrm{C}$, as the $\mathrm{IV}$; other treatments such as blocking the brachial complex, the use of free radical scavengers, botulinum toxin surgical sympathectomy and amputation levels have weak evidence. ${ }^{24}$ According to population studies in the Netherlands only $30 \%$ of patients are considered recovered, 54\% remained stable for five years and $16 \%$ had severe progressive disease..$^{25}$ These data indicate that you have to find another therapeutic option to reduce pain and improve function of the patient.

\section{Imunomodulators}

Cytokines can be divided into interleukins, interferons, hematopoietic colony stimulating factors and tumor necrosis factors. The tumor necrosis factor, $\mathrm{TNFa}$, is a proinflammatory cytokine released by activated macrophages and in response to both an antigen and trigger autoimmune $\mathrm{T}$ lymphocytes. Involved in sequestration of certain cells into inflammatory foci, through the activation of endothelial adhesion molecules and also on the permeability and scarring. To interact with the TNF cells do through specific receptors. The TNFa plays a role in mediating mechanical hyperalgesia in CRPS and other síndromesneuropáticos. Increased levels of interleukins and $\mathrm{TNFa}$ in extremities of these patients suggest an inflammatory process. ${ }^{26}$ Theoretically TNFa inhibition would have a role in treating inflammatory diseases related to inflammatory processes and diseases CRPS. TNFa inhibitors are FDA approved adalimumab, etanercept and infliximab; the most important concepts about them are described below. Adalimumab (Humira) is a human recombinant antiTNFa IgG monoclonal antibody that binds to soluble TNFa and to membranebound TNFa; the result is that neutralizes the biological function by inhibiting its interaction with the p55 and p 75 cell surface TNFa. Also modulates induced or regulated by TNFa level that incluyecambios adhesion molecules which are responsible for leukocyte migration biological response.

Etanercept (Enbrel) is a receptor fusion protein in the TNFa receptor extracellular domain fused to the FC portion of human IgG; by binding it is inhibited to a greater extent the soluble TNFa and TNFa lesser extent bound to the cell membrane. It has two $\mathrm{p} 75$ binding sites, which is why it is very akin to TNFa, rather than the natural receptor Infliximab (Remicade) is a chimeric monoclonal antibody IgG constant antiFNTa fraction consisting of human IgG and murine variable region; it binds to soluble TNFa and to membrane-bound $\mathrm{TNF}$ a thus preventing binding of TNFa to its receptor. Also in vitro has been shown that binds complement and cell apoptosis produces marked with TNFa receptors; thereby signaling receptors prevents the cell surface, and possibly not the cytokine cascade, cell lysis, nor the recruitment of leukocytes, or endothelial activation occurs; so when administered early could prevent changes in central nervous system. ${ }^{27}$ There has been a slight decrease in white blood cells, deviation to the left of lymphocytes, monocytes and neutrophils, without changes in the production of cytokines. It found elevated levels of TNFa in joints of patients with rheumatoid arthritis and psoriatic arthritis; after treatment with infliximab decreased levels of IL 6 and C-reactive protein in blood. In patients with psoriasis I decrease the epidermal inflammation and normalization of keratinocyte differentiation in psoriatic plaques. It is used intravenously at a dose of $3 \mathrm{mg} / \mathrm{kg}$ and reaches dose proportional plasma levels and area under the concentration-time curve; when used in combination with methotrexate concentration increases by $25-30 \%$; distribution in the vascular compartment is mostly and volume of distribution at steady state with a median of 3 to $4.1 \mathrm{lt}$ is independent of dose. The pharmacokinetics is independent of time; the half-life is
8-9 days and is detected in serum for at least 8 weeks after a single infusion with a slight aculacion after the second dose. The clearance and volume of distribution do not change in children when compared to adults, nor to compare the weight; the elimination pathway has not been characterized. Infliximab is indicated for rheumatoid arthritis, Crohn's disease in adults and in children, ulcerative colitis in adults and children, ankylosing spondylitis, psoriatic arthritis and psoriasis. ${ }^{28}$ Contraindications include a history of hypersensitivity to infliximab, to other murine proteins, patients with tuberculosis or other severe infections such as sepsis, abscesses and opportunistic infections and patients with moderate to severe heart failure..$^{28}$

As corticosteroids interactions do not affect significantly the pharmacokinetics; on the contrary the combination of infliximab with other biological therapies, or simultaneous administration of live vaccines or simultaneous administration of therapeutic infectious agents is not recommended. It has been associated with immediate and delayed anaphylactic reactions, possibly by the development of autoantibodies; to minimize these adverse reactions infliximab infusion should be applied in a minimum time period of 2 hours. Other side effects include the emergence of latent infections, abnormal autoimmune reactions and promote the development of lymphomas. One reason for the loss of effectiveness of these treatments is related to the immunogenicity associated, which induces the formation of antibodies and allergic reactions. ${ }^{29}$ Infliximab produces more immunogenic reactions being of mixed origin that etanercept and adalimumab are of human origin. The presence of anti-infliximab in rheumatoid arthritis varies from 12 to $44 \%$, while in Crohn's disease is between 7 and $61 \%$; in these two diseases antibody titres appear to be inversely proporcionalesa to infliximab serum levels and therapeutic response. Regarding the development of antibodies etanercept is 0 to $18 \%$ of patients, and not appear to alter the effectiveness of therapy or the occurrence of adverse reactions. Adalimumab prevalence of antiadalimumab is $1-87 \%$ in rheumatoid arthritis and Crohn's disease, and is associated with a slight worsening of disease control. ${ }^{29}$ The response to treatment depends on the serum concentration of the drug and the presence of antibodies..$^{30}$ The therapeutic ranges have been established in rheumatoid arthritis and are extrapolated to other diseases without clear whether the pharmacokinetics equals in these last and the dose has been established empirically; they must perform measurements of concentrations to establish predictive response models. Treatment changes can be guided by the presence of antibodies; if present is changed to another anti-TNF but are not present should be changed therapeutic target. ${ }^{31}$ Adalimumab problems waking shares infections and tumors. No murine proteins and therefore has less immunogenic reactions; also has the advantage of being administered subcutaneously so that does not depend entirely on the hospital.

\section{Clinical Studies}

A search of infliximab in complex regional pain syndrome database case reports and studies with small numbers of patients were found and reported mixed results. Bernateck has published a case report of successful use in CRPS type 1 by using low-dose infliximab for a regional block. This is a woman with CRPS in the right arm after surgery to correct a Colles fracture and who is Manjo with ibuprofen, steroids, physical therapy and two blocks of stellate ganglion with improved limb temperature without pain relief; It was performed with an intravenous blocking low dose infliximab, $25 \mathrm{mg}$ dissolved in $40 \mathrm{ml}$. of normal saline was repeated one week later. The result was $50 \%$ decrease in pain on the first day and $100 \%$ from the second week, temperature changes of more than 1 degree Celsius, improving the strength and range of motion of hand from the four weeks but 
remains hyperalgesia pressure indicating peripheral sensitization of the nociceptive system persists and pinprick hyperalgesia persisted indicating central sensitization. ${ }^{32}$ Adionalmente this author indicates that treatment with infliximab reduces the local adverse effects and costs. Another study describes the treatment Huygen two CRPS patients who were administered infliximab I intravenously at doses of $3 \mathrm{mg} / \mathrm{kg}$ twice in a period of 4 weeks. Significant decrease local concentrations of IL-6 and TNFa I was found in the limb sick and the contralateral, improved range of motion in 50\%, improved temperature, improved motor skills and pain in $25 \%$ after 4 weeks. ${ }^{33}$

Zijlstra $^{34}$ presents a study assesses response 24 CRPS type 1 patients to intravenous administration of infliximab at a dose of $5 \mathrm{mg} /$ $\mathrm{kg}$ or placebo three times over a period of six weeks. Preliminary reports show normalization of the levels of inflammatory mediators, regional reduction in inflammation and improved quality of life. ${ }^{34}$ More recently Dirckx conducted a double-blind randomized study with 13 patients using infliximab at a dose of $5 \mathrm{mg} / \mathrm{kg}$ in six patients and seven placebo patients, repeating the doses at two and six weeks. Pain reduction was observed but it was not significant, decrease in the level of cytokines greater in the treatment group but not significant and worsening health status in the study group. The study was stopped because I do not get a sufficient number of participants to ensure a sufficient power. Also they described two cases of CRPS after infusions of infliximab. One was a 15 year old patient diagnosed with Crohn's disease, which was recovered after three weeks and se sugiere hipersensibilidad asociada a reaction to infliximab was the trigger of CRPS. ${ }^{35}$ Another was in a patient of 36 years with a diagnosis of ankylosing spondylitis and psoriatic arthritis, which also recovered after three weeks but for which there was no possible explanation for submission..$^{35}$ These studies suggest that the use of infliximab for the treatment of CRPS should be done with caution. Adalimumab case series was conducted with five patients with CRPS type 1 using $40 \mathrm{mg}$ subcutaneous dose in three two-week intervals; observed improvements in pain, depression, and operating parameters but are not significant, and showed only a trend to improve the mechanical thresholds; It should be noted that patients showed better response were in the hot phase of CRPS with temperature differences of 1.5 to 2 degrees between the sick and the healthy limb..$^{36,37}$

\section{Discussion}

One mechanism that may contribute to the emergence of a CRPS is inflammatory, and within this, the important role of TNF a; it has been proposed that by using an anti-TNF antibody such as infliximab the cytokine cascade stops and thus reduce the pain, inflammation and processes of peripheral and central sensitization. He has exhibited in several works effectively decrease proinflammatory cytokines in the affected limb mainly and a little less in the contralateral. Studies with patients corroborate and also show improved range of motion, temperature and pain reduction from 1 day to 4-6 weeks after application; Study shows deterioration in the quality of life and others show persistent phenomena of peripheral and central six months after sensitization. Finally adverse reactions have been described as occurrence of infections, hypersensitivity reactions and two cases of CRPS. Jobs that have generated these resultadoshan been mostly cases and description of two studies, one randomized double blind was suspended because I do not reach a sufficient number of patients. Infliximab has generated great expectations for acting in one of the mechanisms involved in the complex regional pain syndrome and studies presented provide information showing decreased mediators of inflammation and correlate with some components of the clinical picture, but not all, possibly by the participation of other generating mechanisms of CRPS. However the type of published studies and the small number of patients studied do not allow more solid conclusions and the evidence is still low and it is desirable to develop studies to provide further information to help clarify the value of infliximab in this syndrome.

\section{Conclusion}

The role of inflammatory mechanisms in the pathogenesis of CRPS is important and that makes it a therapeutic target that must meet every day. Infliximab is a molecule that has been used in the management of CRPS and have published case reports and two clinical studies showing improvement in range of motion, differences in temperature and decrease in pain persist even phenomena of peripheral and central sensitization after a few months; likewise they described adverse reactions including onset of CRPS in patients who received other necessary conditions and use with caution. The information is so far low level of evidence for such studies from which it was generated so it is necessary to have higher quality studies for more solid conclusions. Likewise, the best results are obtained in the early phases of the disease where the predominant inflammatory component. The information available so far is low level of evidence for such studies, differences in their designs, scales and measuring the low number of patients. The current recommendation for its use is limited so it is necessary to have higher quality studies, to establish higher levels of evidence and to make broader recommendations that manner of use.

\section{Conflicts of Interest}

The authors do not have any personal or financial interests.

\section{Acknowledgments}

None.

\section{Funding}

None.

\section{References}

1. Harden N, Oaklander AL, Burton AW, et al. Complex regional pain syndrome: practical diagnostic and treatment guidelines, 4th edn, Pain Med. (2013);14(2):180-229.

2. Marquez E. Complex regional pain síndrome. Seminars of the Spanish Foundation of Rheumatology. 2012;13(1).

3. De Mos M, AGJ de Bruijnb, Huygenb FJPM, et al. The incidence of complex regional pain syndrome: A population-based study. Pain. (2007);129(1-2):12-20.

4. Sandroni P, Benrud-Larson LM, McClelland RL, et al. Complex regional pain syndrome type I: Incidence and prevalence in Olmsted County, a population-based study. Pain. 2003;103(1-2):199-207.

5. Kiralp MZ, Dincer U, Cakar E, et al. Complex regional pain syndrome: epidemilogic features, treatment approach, workday loss and return to work / disability ratios. Turk J Rheumatol. 2009;24:1-5.

6. Rodriguez RF, Angel AM, Epidemiology and Clinical Characteristics of complex regional pain of upper limb in Valle-Colombia. In press.

7. Albazaz R, Wong YT, Homer-Vanniasinkam S. Complex regional pain syndrome: review article. Ann Vasc Surg. 2008;22(2): 297-306.

8. Bruehl S. An update on the pathofisiology of complex regional pain syndrome. Review article. Anesthesioloy. 2010;113(3):713-725.

9. Oaklander AL, Rissmiller JG, Gelman LB, et al. Evidence of focal small-fiber axonal degeneration in complex regional pain syndrome-I (reflex sympathetic dystrophy). Pain. 2006;120(3):235-243. 
10. PJ Albrecht. Phathologic Alterations of cutaneous innervation and vasculature in Affected limbs from complex regional pain patientes With síyndrome. Pain. 2006;120(3):244-266.

11. Ji RR, Woolf CJ. Neuronal plasticity and signal transduction in nociceptive neurons: Implications for the initiation and maintenance of pathological pain. Neurobiol Dis. 2001;8(1):1-10.

12. Cheng JK, Ji RR. Intracellular signaling in primary sensory neurons and persistent pain. Neurochem Res. 2008;33(10):1970-1978.

13. Janig W. The role of the simpatethic nervous sistema in neuropathic pain: clinical observations and animal models, Neuropatic Pain. Pathophysiology and treatment. IASP Press. 2001.

14. Shurmann M, Gradl G, Zaspel J, et al. Peripheral sympathetic function as a predictor of complex regional pain syndrome tipe I in patients with radial fracture. Auton Neurosci. 2000;86(1-2):127-134.

15. Chemali KR, Gorodeski R, Chelimsky TC. Alpha-adrenergic supersensitivity of the sudomotor nerve in complex regional pain syndrome. Ann Neurol. 2001;49(4):453-459.

16. Juottonen K, Gockel M, Silén T, et al. Altered central sensorimotor processing in patients with complex regional pain syndrome. Pain. 2002;98(3):315-323.

17. Pleger B, Tegenthoff M, Ragert P, et al. Sensorimotor returning in complex regional pain syndrome parallels pain reduction. Ann Neurol. 2005;57(3):425-429.

18. De RooijAM, de Mos M, Sturkenboom MC, et al. Familial occurrence of complex regional pain syndrome. Eur J Pain. 2009;13(2):171-177.

19. De RooijAM, Florencia Gosso M, Haasnoot GW, et al. HLA-B62 and HLA-DQ8 are associated with complex regional pain syndrome with fixed dystonia. Pain. 2009;145(1-2):82-85.

20. Harden RN, Rudin NJ, Bruehl S, et al. Increased systemic catecholamines in complex regional pain syndrome and relationship to phychological factors: A pilot study. Anesth Analg. 2004;99(5):1478-1485.

21. Birkein F. Complex regional pain syndrome. J Neurol. 2005;252:131138.

22. Alexander GM, van Rijn MA, van Hilten JJ, et al. Changes in cerebrospinal fluid levels of proinflammatory cytokines in CRPS. Pain. 2005;116(3):213-219.

23. Schinkel C. Inflammatory mediators are altered in the acute phase of posttraumatic complex regional pain syndrome. Clin J Pain. 2006;22(3):235-239.
24. Salibi A. Complex regional pain syndrome: A sistemic review of the literatura, the past, present and future management. J Pain Relief. 2014;3: 1 .

25. de Mos M, Huygen FJ, van der Hoeven-Borgman M, et al. Outcome of the complex regional pain syndrome. Clin J Pain. 2009;25(7):590-597.

26. Huygen FJ, De Bruijn AG, De Bruin MT, et al. Evidence for local inflammation in complex regional pain syndrome tipe I. Mediators Inflamm. 2002;11(1):47-51

27. Dirckx M, Groeneweg G, Wesseldijk F, et al. Report of a preliminary discontinued doublé-blind, randomized, placebo-controlled trial of the anti-TNF a chimeric monoclonal antibody infliximab in complex regional pain syndrome. Pain pract. 2013;13(8): 633-640.

28. Ficha técnica infliximab. Ema europa eu. Merck.

29. Emi Aikawa N, de Carvalho JF, Artur Almeida Silva C, et al. Inmunogenicitiy of Anti-tnf-Agents in autoinmune diseases. Clinic Rev Allerg Immunol. 2010;38(2-3):82-89.

30. Pascual SD, Plasencia C, Ramiro S, et al. Influence of inmunogenicity on the efficacy of long-term treatment with infliximab in rheumatoid arthritis. Rheumatology (Oxford). 2011;50(8):1445-1452.

31. Bartelds Gm, Wijbrands CA, Nurmohamed MT, et al. Anti-infliximab and anti-adalimumab antibodies in relation to response to adalimumab in infliximab switchers and anti-TNF naive patients: a cohort study. Ann Rheum Dis. 2010;69(5):817-821 .

32. Bernateck M, Rolke R, Birklein F, et al. Successful intravenous regional block with low dose tumor necrosis factor alpha antibody infliximab for treatment of complex regional pain syndrome 1. Anesth Analg. 2007;105(4):1148-1151.

33. Huygen FJ, Niehof S, Zijlstra FJ, et al. Successful treatment of CRPS 1 with anti-TNF. J Pain Symptom Manage. 2004;27(2):101-103.

34. Zijlstra . Successful treatment of CRPS 1 with anti-TNF. J Pain Symptom Manage. 2004;27(2):101-103.

35. Kachko L, Efrat R, Ami SB, Katz J, Mukamel M (2007) Complex regional pain syndrome tipe I after infliximab infusión. Paediatr Anaesth. 2007;17(11):1112-1114.

36. Chahine LM, Patrick R, Tavee J. Complex regional pain syndrome after infliximab infusión. J Pain Letters. 2008;36(3): e2-4.

37. Eisenberg E, Sandler I, Treister R, et al. Anti tumor Necrosis Factoralpha Adalimumab for Complex Regional Pain Syndrome Tipe I: A Case Series. Pain Pract. 2013;13(8):649-656. 